\title{
Endovascular repair of large intercostal artery patch aneurysm using branch stent-graft in a patient with Loeys-Dietz syndrome
}

\author{
Emanuel R. Tenorio, MD, PhD, Tiziano Tallarita, MD, Aleem K. Mirza, MD, Thanila A. Macedo, MD, and
}

Gustavo S. Oderich, MD, Rochester, Minn

\footnotetext{
From the Division of Vascular and Endovascular Surgery, Mayo Clinic, Rochester, Minn.

Disclosures: Dr Oderich has received consulting fees and grants from Cook Medical, W. L. Gore \& Associates, Inc, and GE Healthcare (all paid to Mayo Clinic with no personal income). All other authors have nothing to disclose with regard to commercial support.

Received for publication July 3, 2019; revisions received Aug 8, 2019; accepted for publication Aug 12, 2019; available ahead of print Oct 21, 2019.

Address for reprints: Gustavo S. Oderich, MD, Gonda Vascular Center, Mayo Clinic, 200 First St SW, Rochester, MN 55905 (E-mail: oderich.gustavo@mayo.edu).

J Thorac Cardiovasc Surg 2020;159:e95-9

$0022-5223 / \$ 36.00$

Copyright (C 2019 Published by Elsevier Inc. on behalf of The American Association for Thoracic Surgery https://doi.org/10.1016/j.jtcvs.2019.08.116
}

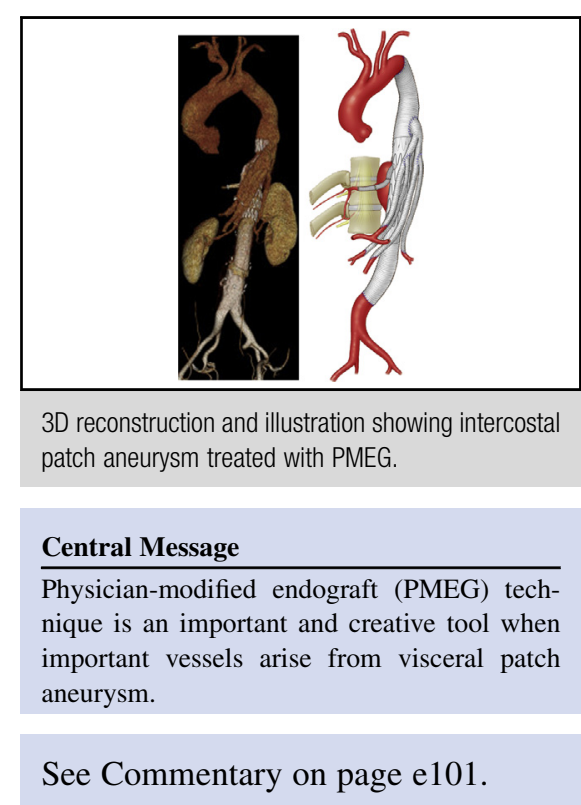

Reimplantation of intercostal and lumbar arteries as an "island" patch is often used during open surgical repair of thoracoabdominal aortic aneurysms (TAAAs) to reduce the risk of spinal cord injury. ${ }^{1}$ The native aorta around the reimplanted vessels is at risk of aneurysmal degeneration, which is more frequent in patients with connective tissue disorders. ${ }^{1-4}$ This report illustrates a patient with Loeys-Dietz syndrome treated who presented with a symptomatic intercostal artery (IA) patch aneurysm following open surgical TAAA repair. The patient was treated urgently by endovascular repair using a physician-modified endograft (PMEG) with a IA directional branch (DB). The patient consented to publication of this case report.

\section{CASE PRESENTATION}

A 28-year-old man with diagnosis of Loeys-Dietz syndrome presented with chest pain and a large IA patch aneurysm 2 years after open surgical repair of Extent II chronic postdissection TAAA (Figure 1). Follow-up consisted of clinical examination, laboratory studies, and imaging before discharge and 2, 6, 12 months, and annually thereafter. The patient was treated with metoprolol and losartan for secondary prevention of aneurysmal degeneration. The patient was treated by endovascular repair using PMEG with a single IA DB.

\section{TECHNIQUE}

\section{Device Modification}

A tapered 36 32- $\times$ 157-mm Cook Zenith TX2 thoracic stent-graft (Cook Medical, Bloomington, Ind) was modified on-site under a strict sterile technique (Figure 2, $A-D$ ). The location of the IA was determined using centerline of flow measurements (TeraRecon, Inc, Foster City, Calif). The stent-graft was unsheathed and one oblique $5 \times 7-\mathrm{mm}$ fenestration was created in the predetermined location using ophthalmic cautery. A $5 \times 25$-mm VIABAHN stentgraft (W. L. Gore \& Associates, Inc, Flagstaff, Ariz) was beveled and anastomosed end-to-side to create a DB using 5-0 running Gore sutures (W. L. Gore \& Associates, Inc). Radiopaque gold markers were placed at the proximal and distal edges of the DB and at 12- and 6-o'clock positions to assist with device orientation during deployment. The DB was preloaded with 0.018-inch V18 (Boston Scientific, Bloomington, Minn) guidewire and resheathed into the original delivery system using 2-0 silk ties and Silastic tape.

\section{Implantation}

A cerebrospinal fluid drain was placed preoperatively (Figure 2, $E-M$ ). The procedure was performed with the patient under general anesthesia with intraoperative 

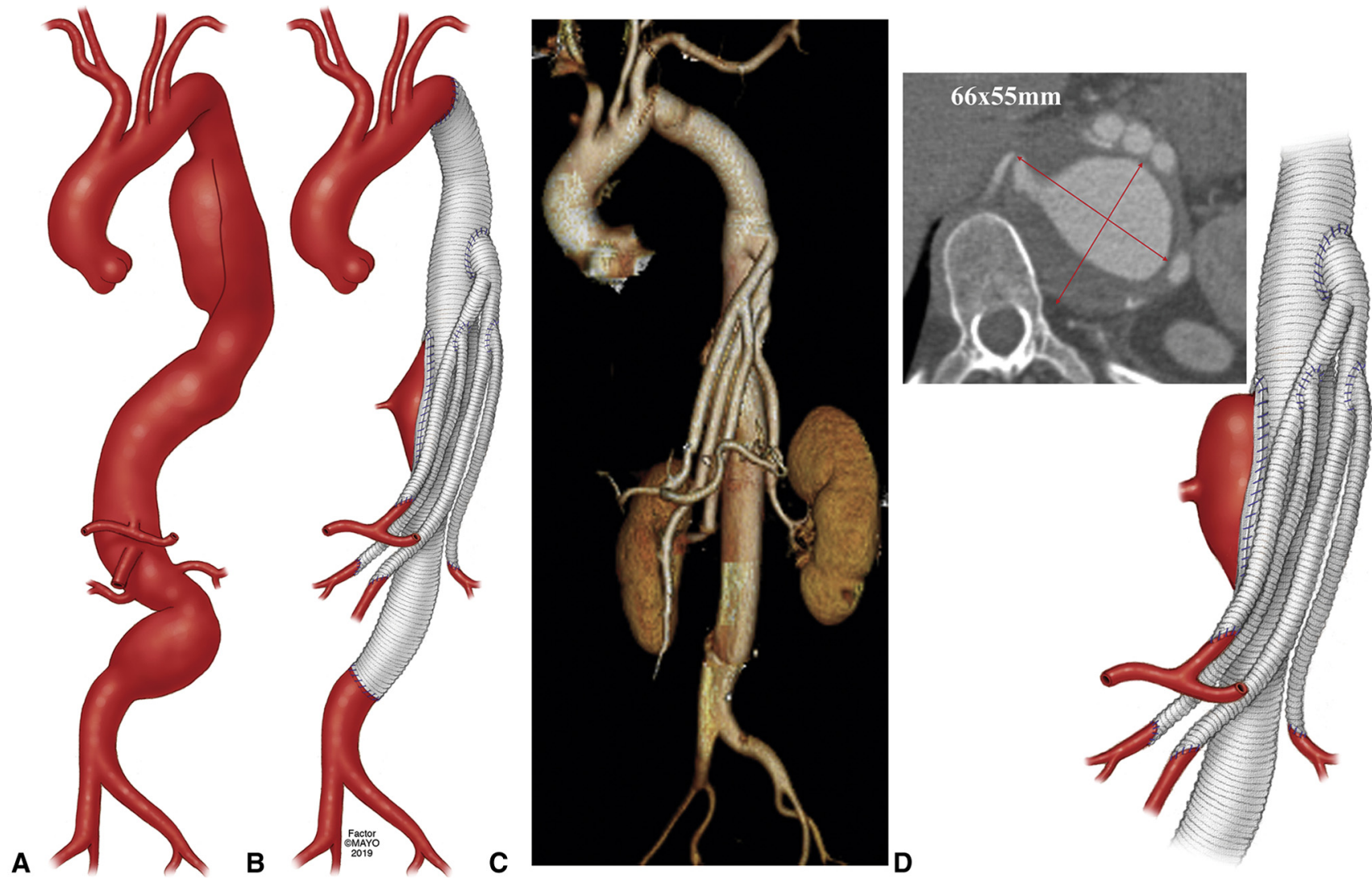

FIGURE 1. Illustration showing an extent II postdissection thoracoabdominal aortic aneurysm (A). Illustration after the open repair showing aortic replacement from left subclavian artery to aortic bifurcation with two 26-mm woven HEMASHIELD grafts, aorta graft to celiac axis and superior mesenteric artery bypass with 18- $\times 9$-mm HEMASHIELD, right and left renal bypass with 8-mm HEMASHIELD, and reimplantation of large intercostal artery (B). Three-dimensional CTA reconstruction showing widely patent all branches (C) and axial view of the CTA and illustration showing the aneurysmal degeneration during the follow-up time with maximum diameter of $66 \mathrm{~mm}$ (D). CTA, Computed tomography angiography. By permission of the Mayo Foundation for Medical Education and Research. All rights reserved.

motor-evoked potential monitoring. The left proximal brachial artery was surgically exposed and percutaneous femoral access was established using a preclosure technique. The patient was heparinized to achieve an activated clotting time $>250$ seconds. A $12-\mathrm{Fr} \times 45-\mathrm{cm}$ DrySeal Flex sheath (W. L. Gore \& Associates, Inc) was introduced via the left brachial approach and positioned in the descending thoracic aorta. A 20-Fr sheath was advanced via the femoral approach into the infrarenal aorta over a 0.035-inch Lunderquist wire (Cook Medical). Throughand-through brachiofemoral access was established, and a 5 -Fr $\times 110-\mathrm{cm}$ sheath was advanced via the brachial access and exteriorized via the right femoral sheath. The femoral sheath was removed over the wire and the 5-Fr brachial sheath while maintaining hemostasis with manual compression on the femoral access site. The PMEG device was loaded into the Lunderquist wire and the preloaded 0.018-inch guidewire into the 5-Fr sheath. The PMEG and preloaded guidewire were introduced via the femoral approach and advanced to the thoracic aorta. The 5-Fr sheath was removed from the brachial access over the preloaded guidewire, allowing an $8 \mathrm{Fr}$ Raabe sheath (Cook Medical) to be advanced over the preloaded guidewire into the descending thoracic aorta. The PMEG was positioned with the distal edge of the DB $2-\mathrm{cm}$ above the level of the target IA. The stent-graft was partially deployed, releasing the DB and allowing advancement of the 8-Fr brachial sheath into the DB over the preloaded 0.018 -inch guidewire. ${ }^{5}$

A 4-Fr Kumpe "buddy" catheter (Cook Medical) was introduced via the 8-Fr sheath and the target IA was successfully catheterized. The stent-graft was fully deployed and a balloon occlusion test was performed using a $5 \times 60-\mathrm{mm}$ angioplasty balloon, which was temporarily inflated in to occlude the DB. After 5 minutes of balloon inflation, there was decline in right lower-limb motor-evoked potentials, with normalization after balloon deflation. The IA was incorporated with placement of $6 \times 50$-mm VIABAHN stent-grafts in the DB. Final angiography showed widely patent stent-graft, DB, and renal-mesenteric bypass grafts (Video 1). Total endovascular time was 125 minutes with fluoroscopy time of 


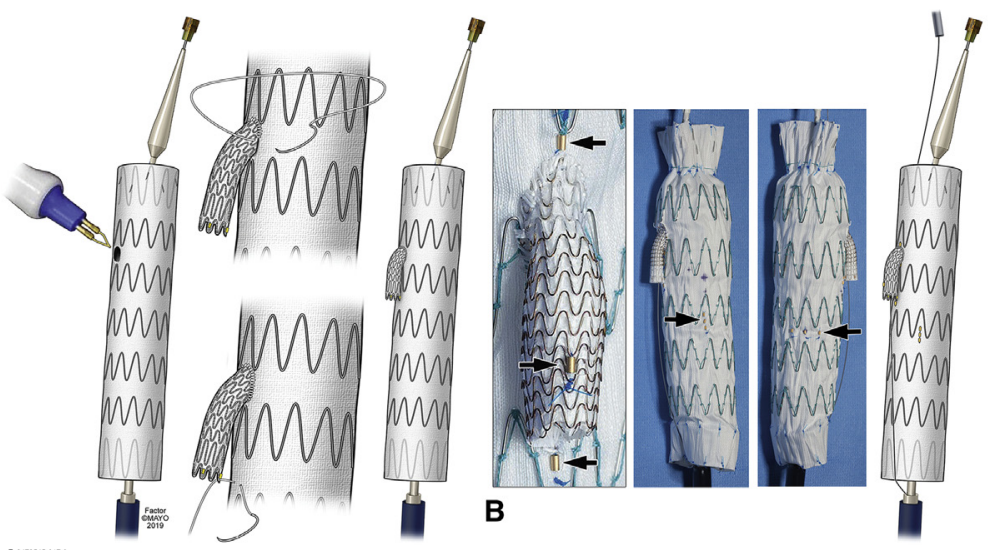

A
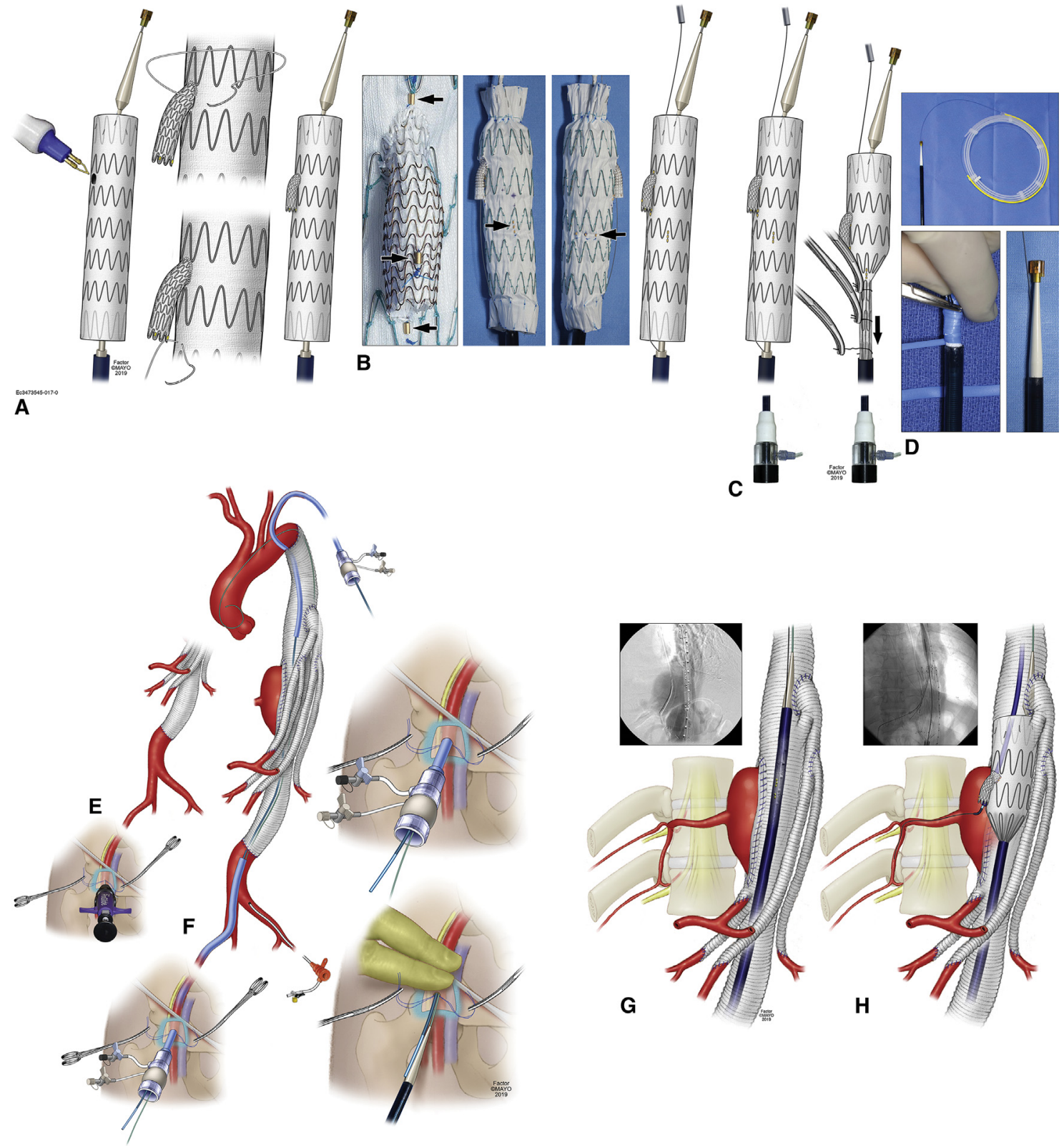

FIGURE 2. Device modification and implantation. The stent-graft was unsheathed and oblique $5 \times 7$-mm fenestration was created in the predetermined location using ophthalmic cautery. A $5 \times 25$-mm VIABAHN was beveled and anastomosed end-to-side to create a DB using 5-0 running Gore sutures (A). The radiopaque gold markers (arrows) around the branch and at 12 and 6 o'clock of the stent-graft for anteroposterior orientation (B). The DB was preloaded with 0.018-inch V18 guidewire and resheathed into the original delivery system using 2-0 silk ties and Silastic tape (C and D). The left proximal brachial artery was surgically exposed and percutaneous femoral access was established using a preclosure technique (E). A 20-Fr sheath was advanced via the femoral approach into the infrarenal aorta over a 0.035-inch Lunderquist wire. Through-and-through brachiofemoral access was established and a 5 -Fr $\times 110$-cm sheath was advanced via the brachial access and exteriorized via the right femoral sheath. The femoral sheath was removed over the wire and the 5-Fr brachial sheath while maintaining hemostasis with manual compression on the femoral access site. The device was loaded into the Lunderquist wire and the preloaded 0.018-inch guidewire into the 5-Fr sheath (F). The device was positioning with the distal edge of the DB $2 \mathrm{~cm}$ above the level of the target IA $(\mathrm{G})$. The device was partially deployment and the IA was catheterized $(\mathrm{H})$. The stent-graft was fully deployed and a balloon occlusion test was performed using a $5 \times 60$ - $\mathrm{mm}$ angioplasty balloon, which was temporarily inflated in to occlude the DB. After 5 minutes of balloon inflation, there was decline in right lower-limb motor-evoked potentials, with normalization after balloon deflation (I). The IA was incorporated with placement of two $6 \times 50$-mm VIABAHN stent-grafts in the DB ( $\mathrm{J}$ and $\mathrm{K}$ ). Final angiography showed widely patent stent-graft, DB and renal-mesenteric bypass grafts $(\mathrm{L})$. Three-dimensional computed tomography angiography reconstruction and illustration showing the intercostal branch artery patent after 4 years' follow-up with aneurysm sac shrink (M). DB, Directional branch; $I A$, intercostal artery; Ham, hamstring; AT, anterior tibial; $A H$, abductor hallucis. By permission of Mayo Foundation for Medical Education and Research. All rights reserved. 

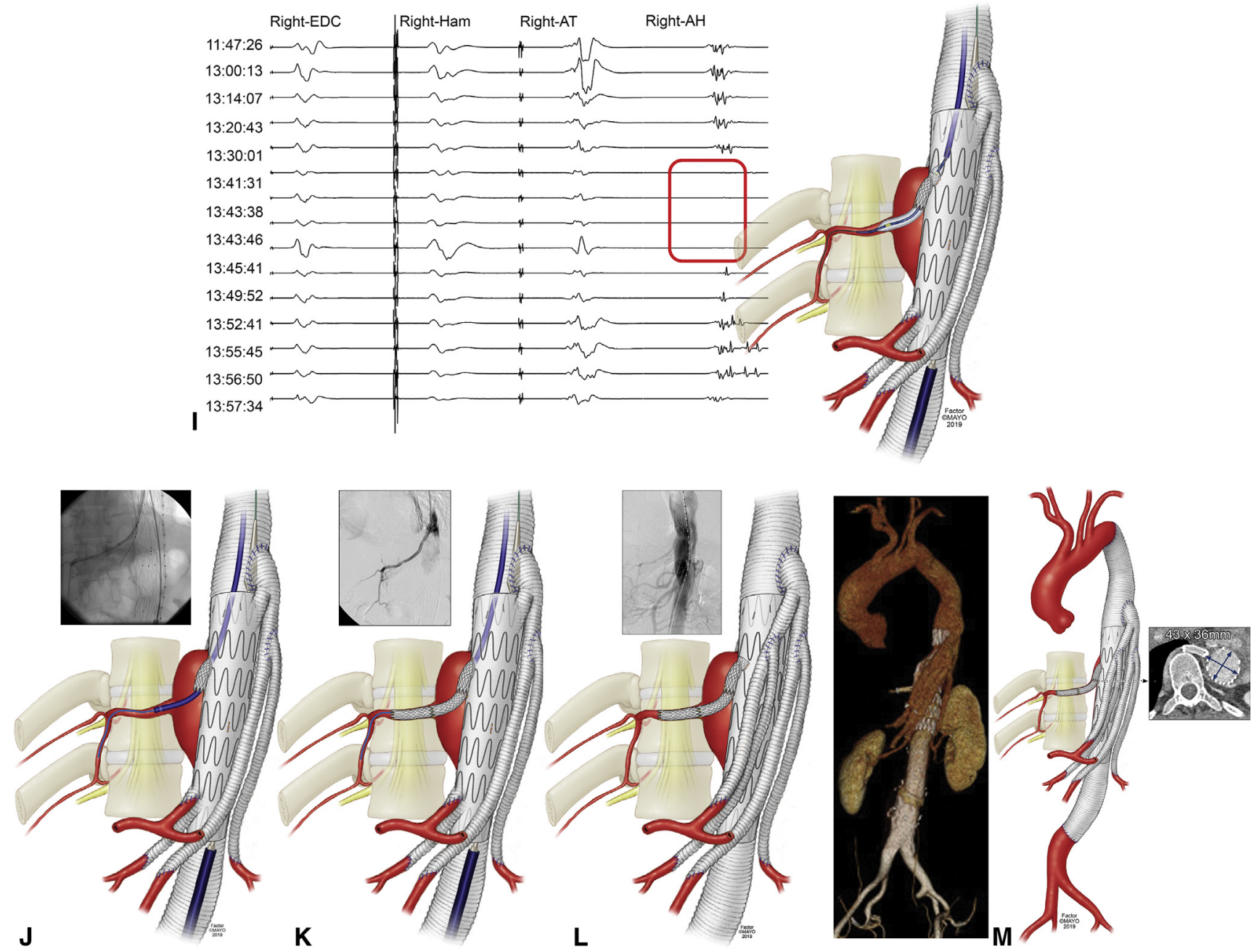

FIGURE 2. (Continued)

31 minutes. Total contrast used was $70 \mathrm{~mL}$. The cerebrospinal fluid drain was removed on postoperative day 2 , and the patient was discharged home on postoperative day 4 with no complications. At 4 years, the patient had no complications, and computed tomography angiography showed patent DB with complete remodeling of the IA patch aneurysm.

\section{DISCUSSION}

Endovascular incorporation of IAs is challenging because of the small, tortuous anatomy and posterior location. This case illustrates the technical success and mid-term durability of IA DB using the PMEG technique in a patient with Loeys-Dietz syndrome. The technique was possible because of large diameter of the IA and presence of surgical grafts as landing zones of the aortic branched stent-graft, thus limiting the risk of type I endoleak.

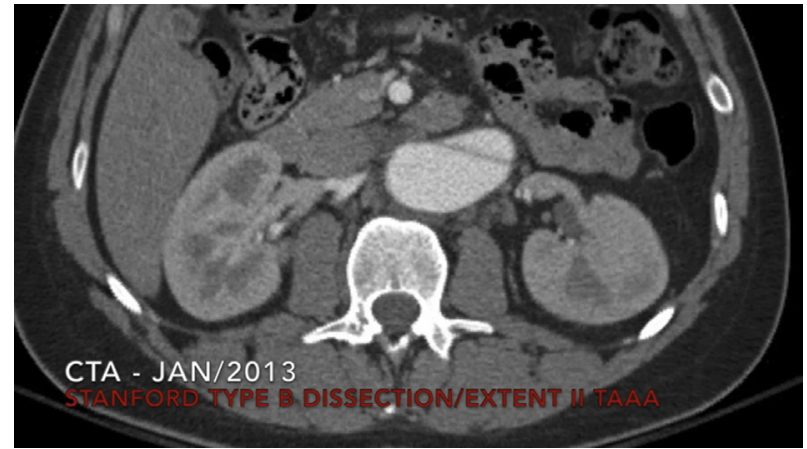

VIDEO 1. Intercostal artery patch aneurysm treated using physicianmodified technique to create a branch to preserve the flow into the intercostal artery. By permission of Mayo Foundation for Medical Education and Research. All rights reserved. Video available at: https://www.jtcvs. org/article/S0022-5223(19)31928-2/fulltext. 
The prevalence of visceral patch aneurysm ranges from $1.1 \%$ to $7.7 \%$, with 3 -fold greater prevalence in patients with connective tissue disorders. ${ }^{3,6,7}$ We recommend intervention for aneurysms that are saccular and expanding with diameter of 5 to $6 \mathrm{~cm}$, or in those that are symptomatic or have rapid expansion. Imaging surveillance of open TAAA repair is important, particularly in younger patients with connective tissue disorders and in those reconstructed with island patches. This is done with computed tomography angiography or magnetic resonance yearly.

\section{References}

1. Crawford ES, Snyder DM, Cho GC, Roehm JO Jr. Progress in treatment of thoracoabdominal and abdominal aortic aneurysms involving celiac, superior mesenteric, and renal arteries. Ann Surg. 1978;188:404.
2. Adam DJ, Berce M, Hartley DE, Robinson DA, Anderson JL. Repair of recurren visceral aortic patch aneurysm after thoracoabdominal aortic aneurysm repair with a branched endovascular stent graft. J Vasc Surg. 2007;45:183-5.

3. Bertoglio L, Mascia D, Cambiaghi T, Kahlberg A, Tshomba Y, Gomez JC, et al. Management of visceral aortic patch aneurysms after thoracoabdomina repair with open, hybrid, or endovascular approach. J Vasc Surg. 2018;67: 1360-71.

4. Afifi RO, Sandhu HK, Trott AE, Nguyen TC, Miller CC, Estrera AL, et al. Redo thoracoabdominal aortic aneurysm repair: a single-center experience over 25 years. Ann Thorac Surg. 2017;103:1421-8.

5. Oderich GS, Mendes BC, Correa MP. Preloaded guidewires to facilitate endovascular repair of thoracoabdominal aortic aneurysm using a physician-modified branched stent graft. J Vasc Surg. 2014;59:1168-73.

6. Dardik A, Perler BA, Roseborough GS, Williams GM. Aneurysmal expansion of the visceral patch after thoracoabdominal aortic replacement: an argument for limiting patch size? J Vasc Surg. 2001;34:405-10.

7. Kulik A, Allen BT, Kouchoukos NT. Incidence and management of intercostal patch aneurysms after repair of thoracoabdominal aortic aneurysms. J Thorac Cardiovasc Surg. 2009;138:352-8. 